\title{
Fixed point approach to Basset problem
}

\author{
Lale Cona \\ Department of Mathematical Engineering, Gumushane University, Gumushane, Turkey
}

Received: 31 January 2017, Accepted: 7 February 2017

Published online: 25 August 2017.

\begin{abstract}
In the present paper, a sufficient condition for existence and uniqueness of Basset problem is obtained. The theorem on existence and uniqueness is established. This approach permits us to use fixed point iteration method to solve problem for differential equation involving derivatives of nonlinear order.
\end{abstract}

Keywords: Fixed Point, initial Value Problem, Basset equation, Riemann-Liouville derivative.

\section{Introduction}

It is well known that differential equations involving derivatives of non-integer order are used in modelling of various physical phenomena in areas like diffusion processes, damping laws, etc. (see [1]-[7]). Methods of solutions of problems for fractional differential equations have been studied extensively by many researchers (see [1]-[12] and references therein).

Let us give definitions of fractional derivatives and fractional powers of positive operators that will be needed below [11].

Definition 1. If $x(t) \in C([a, b])$ and $a<x<b$, then

$$
I_{a+}^{\alpha} x(t):=\frac{1}{\Gamma(\alpha)} \int_{a}^{t} \frac{x(t)}{(t-s)^{1-\alpha}} d t,
$$

where $\alpha \in(-\infty, \infty)$ is called the Riemann-Liouville fractional integral of order $\alpha$. In the same manner for $\alpha \in(0,1)$

$$
D_{a+}^{\alpha} x(t):=\frac{1}{\Gamma(1-\alpha)} \frac{d}{d t} \int_{a}^{t} \frac{x(s)}{(t-s)^{\alpha}} d s,
$$

is called the Riemann-Liouville fractional derivative of order $\alpha$.

Note that if $x(a)=0$, then we can write

$$
D_{a+}^{\alpha} x(t):=\frac{1}{\Gamma(1-\alpha)} \int_{a}^{t} \frac{x^{\prime}(s)}{(t-s)^{\alpha}} d s .
$$

Here,

$$
\Gamma(\alpha)=\int_{0}^{\infty} s^{\alpha-1} e^{-s} d s \quad(\alpha>0)
$$


Consider initial value problem for Basset equation

$$
\left\{\begin{array}{l}
\frac{d x(t)}{d t}+D_{a+}^{\alpha} x(t)=f(t, x(t)), 0<t<T \\
0<\alpha<1 \\
x(0)=\varphi
\end{array}\right.
$$

Actually, fractional differential equation corresponds to the Basset problem [3].

We now shortly describe the organization of the paper. In section 2, we give the basic definitions of fixed point, contraction and the basic concepts we need. In section 3 , we obtain a sufficient condition for existence and uniqueness of problem and establish the theorem on existence and uniqueness.

\section{Fixed point and contraction}

Definition 2. Let $E=(E, d)$ be a metric space. A fixed point of a mapping $A: E \longrightarrow E$ of set $E$ into itself is an element $x \in E$ which is mapped onto itself, that is, Ax $=x$, the image Ax coincides with $x$.

Definition 3. A maping $A: E \longrightarrow E$ is called a contraction on $E$, if there is a positive real number $\alpha<1$ such that for all $x, y \in E$

$$
d(A x, A y) \leq \alpha d(x, y)
$$

Now, we state the existence uniqueness theorem the most important application of the Fixed-Point Theorem to ordinary differential equations. We will consider the initial value problem of the form

$$
\frac{d x(t)}{d t}=f(t, x(t)),\left|t-t_{0}\right| \leq a, x\left(t_{0}\right)=x_{0}
$$

The problem for ordinary differential equations will be converted to an integral equation, which defines a mapping $A$, and the conditions of the theorem will imply that $A$ is a contraction such that its fixed point becomes the solution of problem.

Theorem 1. [13] Assume that $f$ is continuous on the rectangle

$$
D=\left\{(t, x):\left|t-t_{0}\right| \leq a,\left|x-x_{0}\right| \leq b\right\}
$$

and thus bounded on $D$, i.e,

$$
|f(t, x)| \leq c \text { for all }(t, x) \in D
$$

Suppose that $f$ satisfies a Lipschitz condition on D with respect to its second argument, that is, there is a constant $l$ such that for $(t, x),(t, y) \in D$

$$
|f(t, x)-f(t, y)| \leq l|x-y|
$$

Then, initial value problem has a unique solution $x$ defined on $\left|t-t_{0}\right| \leq \beta$, where

$$
\beta<\min \left\{a, \frac{b}{c}, \frac{1}{l}\right\} \text {. }
$$

This function $x$ is the limit of iterative sequence $\left\{x_{n}\right\}_{n=0}^{\infty}$ defined by the recursive Picard iteration formula

$$
x_{n}(t)=x_{0}+\int_{t_{0}}^{t} f\left(s, x_{n-1}(s)\right) d s, n \in \mathbb{N}
$$

(C) 2017 BISKA Bilisim Technology 
where $x_{0}(t)$ is an arbitrary continuous function. Error bounds are

$$
\begin{gathered}
d\left(x_{n}, x\right) \leq \frac{\alpha^{n}}{1-\alpha} e^{2 \alpha L} d\left(x_{0}, x_{1}\right), \\
d\left(x_{n}, x\right) \leq \frac{\alpha}{1-\alpha} e^{2 \alpha L} d\left(x_{n-1}, x_{n}\right), n \in \mathbb{N},
\end{gathered}
$$

where $\alpha=l \beta$.

\section{Main results}

Firstly, we defined the metric $d$ on $C_{[0, T]}^{1}$ be the complete space of all continuously differentiable functions defined on the interval $[0, T]$.

Definition 4. Let $C_{[0, T]}^{1}$ be the complete space of all continuously differentiable functions defined on the interval $[0, T]$ with the metric $d$ defined by

$$
d(x, y)=\max _{t \in[0, T]}|x(t)-y(t)|+\max _{t \in[0, T]}\left|\frac{d x(t)}{d t}-\frac{d y(t)}{d t}\right| .
$$

Now, we consider the following initial value problem for Basset equation

$$
\frac{d x(t)}{d t}+D_{0+}^{\alpha} x(t)=f(t, x(t)), 0<t<T, 0<\alpha<1, x(0)=\varphi .
$$

In that case below theorem, we give sufficient condition for existence and uniqueness of this problem.

Theorem 2. Letf be continuous function on the rectangle

$$
B=\{(t, x): t \in[0, T]\} \subseteq \mathbb{R}^{2},
$$

and $|f(t, x)| \leq c$ for all $(t, x) \in B$. Moreover, $f$ satisfies a Lipschitz condition on $B$ with respect to its second argument, i.e, there is a positive constant $l$ such that for arbitrary $(t, x),(t, y) \in B$

$$
|f(t, x)-f(t, y)| \leq l|x-y|
$$

is valid. Moreover, let

$$
g(\alpha, T, l)=\frac{T^{-\alpha+2}}{\Gamma(1-\alpha)(-\alpha+1)(-\alpha+2)}+T l
$$

and suppose that

$$
g(\alpha, T, l)<1 .
$$

Then, Basset problem (3) has a unique solution $x \in C_{[0, T]}$.

Proof. By integrating both sides of Basset equation (3),

$$
\begin{gathered}
\int_{0}^{t} \frac{d x(p)}{d t} d p+\int_{0}^{t} D_{0}^{\alpha} x(p) d p=\int_{0}^{t} f(p, x(p)) d p \\
\left.x(p)\right|_{0} ^{t}+\int_{0}^{t} D_{0}^{\alpha} x(p) d p=\int_{0}^{t} f(p, x(p)) d p
\end{gathered}
$$




$$
\begin{gathered}
x(t)-x(0)+\int_{0}^{t} D_{0}^{\alpha} x(p) d p=\int_{0}^{t} f(p, x(p)) d p \\
x(t)=-\int_{0}^{t}\left(\frac{1}{\Gamma(1-\alpha)} \int_{0}^{p} \frac{x^{\prime}(s)}{(p-s)^{\alpha}} d s\right) d p+\int_{0}^{t} f(p, x(p)) d p+\varphi .
\end{gathered}
$$

Then we obtain integral equation,

$$
x(t)=-\frac{1}{\Gamma(1-\alpha)} \int_{0}^{t} \int_{0}^{p}(p-s)^{-\alpha} x^{\prime}(s) d s d p+\int_{0}^{t} f(p, x(p)) d p+\varphi .
$$

This function $x$ is the limit of the iterative sequence $\left\{x_{n}\right\}_{n=0}^{\infty}$ defined by the recursive Picard iteration formula

$$
x_{n}(t)=-\frac{1}{\Gamma(1-\alpha)} \int_{0}^{t} \int_{0}^{p}(p-s)^{-\alpha} x_{n-1}^{\prime}(s) d s d p+\int_{0}^{t} f\left(p, x_{n-1}(p)\right) d p+\varphi, n \in \mathbb{N}
$$

where $x_{0}(t)$ is an arbitrary continuous function. Error bounds are

$$
\begin{gathered}
d\left(x_{n}, x\right) \leq \frac{g^{n}}{1-g} e^{g L} d\left(x_{0}, x_{1}\right), \\
d\left(x_{n}, x\right) \leq \frac{g}{1-g} e^{g L} d\left(x_{n-1}, x_{n}\right), n \in \mathbb{N}
\end{gathered}
$$

where $\alpha=\frac{l}{L}$. Here, $L$ is a fixed number such that $L>l$. We see that initial value problem (3) can be written in the equivalent integral form (6), which is in the form $x=A x$, where $A: C_{[0, T]}^{1} \longrightarrow C_{[0, T]}^{1}$ is an operator defined by

$$
A x(t)=-\frac{1}{\Gamma(1-\alpha)} \int_{0}^{t} \int_{0}^{p}(p-s)^{-\alpha} x^{\prime}(s) d s d p+\int_{0}^{t} f(p, x(p)) d p+\varphi,
$$

where $f$ is continuous function on the rectangle $B$. Since,

$$
A x(t)=-\frac{1}{\Gamma(1-\alpha)} \int_{0}^{t} \int_{0}^{p}(p-s)^{-\alpha} x^{\prime}(s) d s d p+\int_{0}^{t} f(p, x(p)) d p+\varphi
$$

under assumptions of theorem, by using (2), we find

$$
\begin{aligned}
|A x(t)| & =\left|-\frac{1}{\Gamma(1-\alpha)} \int_{0}^{t}\left(\int_{0}^{p}(p-s)^{-\alpha} x^{\prime}(s) d s\right) d p+\int_{0}^{t} f(p, x(p)) d p+\varphi\right| \\
& \leq \frac{1}{|\Gamma(1-\alpha)|}\left|\int_{0}^{t} \int_{0}^{p}(p-s)^{-\alpha} x^{\prime}(s) d s d p\right|+\left|\int_{0}^{t} f(p, x(p)) d p\right|+|\varphi| \\
& \leq \frac{1}{\Gamma(1-\alpha)} \int_{0}^{t}\left(\int_{0}^{p}(p-s)^{-\alpha} x^{\prime}(s) d s\right) d p+\int_{0}^{t}|f(p, x(p))| d p+|\varphi|
\end{aligned}
$$




$$
\begin{aligned}
& \leq \frac{1}{\Gamma(1-\alpha)} \int_{0}^{t}\left(\int_{0}^{p}(p-s)^{-\alpha} \max _{s \in[0, T]}\left|x^{\prime}(s)\right| d s\right) d p+\int_{0}^{t} c d p+|\varphi| \\
& =\frac{\max _{s \in[0, T]}\left|x^{\prime}(s)\right|}{\Gamma(1-\alpha)} \int_{0}^{t}\left(\left.\frac{-(p-s)^{-\alpha+1}}{-\alpha+1}\right|_{0} ^{p}\right) d p+c t+|\varphi| \\
& =\frac{\max _{s \in[0, T]}\left|x^{\prime}(s)\right|}{\Gamma(1-\alpha)}\left(\int_{0}^{t} \frac{p^{-\alpha+1}}{-\alpha+1} d p\right)+c t+|\varphi| \\
& =\frac{\max _{s \in[0, T]}\left|x^{\prime}(s)\right|}{\Gamma(1-\alpha)}\left(\left.\frac{p^{-\alpha+2}}{(-\alpha+1)(-\alpha+2)}\right|_{0} ^{t}\right)+c t+|\varphi| \\
& =\frac{\max _{s \in[0, T]}\left|x^{\prime}(s)\right|}{\Gamma(1-\alpha)} \frac{t^{-\alpha+2}}{(-\alpha+1)(-\alpha+2)}+c t+|\varphi| .
\end{aligned}
$$

Thus, we have show that $A x \in C^{1}[0, T]$ if $x \in C^{1}[0, T]$; i.e., $A$ maps the set $C^{1}[0, T]$ itself. Now, we show that, $A$ is a contraction map on $C_{[0, T]}^{1}$. Applying the Lipschitz condition (4), we get

$$
\begin{aligned}
|A x(t)-A y(t)| & =\mid-\frac{1}{\Gamma(1-\alpha)} \int_{0}^{t} \int_{0}^{p}(p-s)^{-\alpha} x^{\prime}(s) d s d p+\int_{0}^{t} f(p, x(p)) d p \\
& +\frac{1}{\Gamma(1-\alpha)} \int_{0}^{t} \int_{0}^{p}(p-s)^{-\alpha} y^{\prime}(s) d s d p-\int_{0}^{t} f(p, y(p)) d p \mid \\
& \leq \frac{1}{\Gamma(1-\alpha)}\left|\int_{0}^{t} \int_{0}^{p}(p-s)^{-\alpha}\left(x^{\prime}(s)-y^{\prime}(s)\right) d s d p\right|+\int_{0}^{t}|f(p, x(p))-f(p, y(p))| d p \\
& \leq \frac{1}{\Gamma(1-\alpha)} \max _{s \in[0, T]}\left|x^{\prime}(s)-y^{\prime}(s)\right| \int_{0}^{t} \int_{0}^{p}(p-s)^{-\alpha} d s d p+t l|x(s)-y(s)| \\
& =\frac{1}{\Gamma(1-\alpha)} \max _{s \in[0, T]}\left|x^{\prime}(s)-y^{\prime}(s)\right| \frac{t^{-\alpha+2}}{(-\alpha+1)(-\alpha+2)}+t l|x(s)-y(s)| \\
& \leq \frac{t^{-\alpha+2}}{\Gamma(1-\alpha)(-\alpha+1)(-\alpha+2)} d(x, y)+t l d(x, y) \\
& =\left(\frac{t^{-\alpha+2}}{\Gamma(1-\alpha)(-\alpha+1)(-\alpha+2)}+t l\right) d(x, y) .
\end{aligned}
$$

Taking maximum from both sides we have

$$
\begin{aligned}
d(A x, A y) & \leq \max _{t \in[0, T]}\left(\frac{t^{-\alpha+2}}{\Gamma(1-\alpha)(-\alpha+1)(-\alpha+2)}+t l\right) d(x, y) \\
& =\left(\frac{T^{-\alpha+2}}{\Gamma(1-\alpha)(-\alpha+1)(-\alpha+2)}+T l\right) d(x, y) \\
& \leq g(\alpha, T, l) d(x, y)
\end{aligned}
$$

From (4) we see that $g(\alpha, T, l)<1$, so

$$
d(A x, A y) \leq d(x, y) .
$$


Thus, $A$ is a contraction on $C_{[0, T]}^{1}$. Therefore, $A$ has a unique fixed point $x \in C_{[0, T]}^{1}$, that is, a continuous function on $[0, T]$ satisfying $x=A x$. By (7), we have

$$
x(t)=-\frac{1}{\Gamma(1-\alpha)} \int_{0}^{t} \int_{0}^{p}(p-s)^{-\alpha} x^{\prime}(s) d s d p+\int_{0}^{t} f(p, x(p)) d p+\varphi .
$$

Example 1.Solve initial value problem for Basset equation

$$
\frac{d x(t)}{d t}+D_{0+}^{\alpha} x(t)=1+\frac{2}{\sqrt{\pi}} \sqrt{x(t)}, 0<t<T, x(0)=0
$$

by the iteration method.

Solution 1.By integrating both sides of Basset equation (10),

$$
x(t)=-\frac{1}{\sqrt{\pi}} \int_{0}^{t} \int_{0}^{p}(p-s)^{-\alpha} x^{\prime}(s) d s d p+\int_{0}^{t}\left(1+\frac{2}{\sqrt{\pi}} \sqrt{x(p)}\right) d p
$$

Since $f(t, x(t))=1+\frac{2}{\sqrt{\pi}} \sqrt{x(t)}, 0<t<T, \alpha=\frac{1}{2}, \varphi=0$, we have that $l=\frac{1}{\sqrt{\pi}}$. Let $x_{0}(t)=0$. Now, $x_{n}(t)$ is defined by formula

$$
x_{n}(t)=-\frac{1}{\Gamma(1-\alpha)} \int_{0}^{t} \int_{0}^{p}(p-s)^{-\alpha} x_{n-1}^{\prime}(s) d s d p+\int_{0}^{t} f\left(p, x_{n-1}(p)\right) d p+\varphi, \quad n \in \mathbb{N}
$$

Then,

$$
\begin{aligned}
& x_{1}(t)=-\frac{1}{\Gamma\left(\frac{1}{2}\right)} \int_{0}^{t} \int_{0}^{p}(p-s)^{-\frac{1}{2}} x_{0}^{\prime}(s) d s d p+\int_{0}^{t} f\left(p, x_{0}(p)\right) d p+\varphi \\
& =-\frac{1}{\sqrt{\pi}} \int_{0}^{t} \int_{0}^{p}(p-s)^{-\frac{1}{2}} 0 d s d p+\int_{0}^{t}\left(1+\frac{2}{\sqrt{\pi}} \sqrt{0}\right) d p+0=t, \\
& x_{2}(t)=-\frac{1}{\Gamma\left(\frac{1}{2}\right)} \int_{0}^{t} \int_{0}^{p}(p-s)^{-\frac{1}{2}} x_{1}^{\prime}(s) d s d p+\int_{0}^{t} f\left(p, x_{1}(p)\right) d p+\varphi \\
& =-\frac{1}{\sqrt{\pi}} \int_{0}^{t} \int_{0}^{p}(p-s)^{-\frac{1}{2}} d s d p+\int_{0}^{t}\left(1+\frac{2}{\sqrt{\pi}} p^{\frac{1}{2}}\right) d p \\
& =-\frac{1}{\sqrt{\pi}} \int_{0}^{t}\left(-\left.2(p-s)^{\frac{1}{2}}\right|_{0} ^{p}\right) d p+\left.\left(p+\frac{2}{\sqrt{\pi}} \frac{p^{\frac{3}{2}}}{\frac{3}{2}}\right)\right|_{0} ^{t}=t \text {. } \\
& x_{3}(t)=-\frac{1}{\Gamma\left(\frac{1}{2}\right)} \int_{0}^{t} \int_{0}^{p}(p-s)^{-\frac{1}{2}} x_{2}^{\prime}(s) d s d p+\int_{0}^{t} f\left(p, x_{2}(p)\right) d p+\varphi \\
& =\frac{1}{\sqrt{\pi}} \int_{0}^{t} \int_{0}^{p}(p-s)^{-\frac{1}{2}} d s d p+\int_{0}^{t}\left(1+\frac{2}{\sqrt{\pi}} p^{\frac{1}{2}}\right) d p=t .
\end{aligned}
$$

(C) 2017 BISKA Bilisim Technology 
In similar manner, it can be showed

$$
x_{n}(t)=t, \quad n \in \mathbb{N}
$$

Hence,

$$
x(t)=\lim _{n \longrightarrow \infty} x_{n}(t)=\lim _{n \longrightarrow \infty} t=t .
$$

\section{Conclusion}

In this work, we consider initial value problem for Basset equation. We obtain a sufficient condition for existence and uniqueness of this problem and establish the theorem on existence and uniqueness. This approach permits us to use fixed point iteration method to solve problem for differential equation involving derivatives of nonlinear order.

\section{Competing interests}

The authors declare that they have no competing interests.

\section{Authors' contributions}

All authors have contributed to all parts of the article. All authors read and approved the final manuscript.

\section{References}

[1] Podlubny I: Mathematics in Science and Engineering 198. In Fractional Differential Equations. Academic Press, San Diego; 1999.

[2] Samko SG, Kilbas AA, Marichev OI: Fractional Integrals and Derivatives. Gordon \& Breach, Yverdon; 1993.

[3] Basset AB: On the descent of a sphere in a viscous liquid. Q. J. Math. 1910, 42: 369-381.

[4] Ashyralyev A: Well-posedness of the Basset problem in spaces of smooth functions. Appl. Math. Lett. 2011, 24: 1176-1180. 10.1016/j.aml.2011.02.002

[5] Ashyralyev A, Well-posedness of fractional parabolic equations, Boundary Value Problems, 2013: 2013:31, 1-18.

[6] Baleanu D, Garra, R, Petras, I: A Fractional Variational Approach to the Fractional Basset-Type Equation, Reports on Mathematical Physics, 72 (1) 57-64, 2013.

[7] Petras I: Fractional-Order Nonlinear Systems: Modeling, Analysis and Simulation, Springer, New York (2011)

[8] Cakir Z: Stability of difference schemes for fractional parabolic PDE with the Dirichlet-Neumann conditions. Abstr. Appl. Anal. 2012., 2012: Article ID 463746

[9] Ashyralyev A, Cakir Z: On the numerical solution of fractional parabolic partial differential equations with the Dirichlet condition. Discrete Dyn. Nat. Soc. 2012., 2012: Article ID 696179.

[10] Ashyralyev A. , Z. Cakir; FDM for fractional parabolic equations with the Neumann condition, Advances in Difference Equations, Vol. 2013, No. 120, doi:10.1186/1687-1847-2013-120, 2013.

[11] Ashyralyev A., A note on fractional derivatives and fractional powers of operators. JMAA, 357(2009) 232-236.

[12] Ashyralyev A., N. Emirov, Z. Cakir; Well-posedness of fractional parabolic differential and difference equations with DirchletNeumann condition, Electronic Journal of Differential Equations, Vol. 2014, No. 97, pp. 1—17, 2014.

[13] Erwin Kreyszig, Advanced Engineering Mathematics, John Willey \& Sons, New York,1993. 